ANNALES

POLONICI MATHEMATICI

$83.1(2004)$

\title{
The Picard-Ionescu problem for hyperbolic inclusions with modified argument
}

by Georgeta Teodoru (Iaşi)

\begin{abstract}
We consider the Picard-Ionescu problem for hyperbolic inclusions with modified argument. Existence of a local solution is proved and some properties of the set of solutions are established.
\end{abstract}

1. Introduction. In his $1927 \mathrm{PhD}$ thesis [17], D. V. Ionescu studied, for the first time in the mathematical literature, boundary value problems of Darboux, Cauchy, Picard and Goursat types for second order partial differential equations with modified argument.

More recently, a series of authors studied the same problems for second order hyperbolic equations and inclusions of various forms.

The existence of solutions to the Darboux problem for second order hyperbolic inclusions on bounded domains and unbounded domains in Banach spaces has been studied [1] by several authors by various methods.

For example, Marian Dawidowski, Michał Kisielewicz and Ireneusz $\mathrm{Ku}-$ biaczyk [9] consider the Darboux problem

$$
\left\{\begin{array}{l}
z_{x y}^{\prime \prime} \in F(x, y, z) \quad \text { for a.e. }(x, y) \in P=[0, a] \times[0, b] \\
z(x, 0)=u(x), \quad z(0, y)=v(y), \quad \text { for } x \in[0, a] \text { and } y \in[0, b], \\
z(0,0)=v(0)=u(0)=z_{0},
\end{array}\right.
$$

where $F: P \times E \rightarrow 2^{E}$ is a multifunction with nonempty values and $E$ is a separable Banach space.

Assuming that:

(A) $F(x, y, \cdot): E \rightarrow 2^{E}$ is lower-semicontinuous for each fixed $(x, y) \in P$;

(B) $F$ is measurable on $P \times E$,

2000 Mathematics Subject Classification: 35L15, 35R70.

Key words and phrases: multifunction, hyperbolic inclusion, upper-semicontinuity, initial values, function absolutely continuous in Carathéodory's sense. 
and other hypotheses, the existence of a Carathéodory selection for $F$ being ensured, the authors prove, by the Artstein-Prikry selection theorem, the existence of a solution in the domain $P$.

The Darboux, Cauchy, Picard and Goursat problems for the same inclusion were studied by Georgeta Teodoru in [26] and in a series of papers, under the hypothesis that $F(x, y, \cdot): \Omega \rightarrow 2^{\mathbb{R}^{n}}$, where $\Omega$ is an open subset in $\mathbb{R}^{n}$, is upper-semicontinuous, using the Kakutani-Ky Fan fixed point theorem.

Several authors have investigated the Darboux problem for hyperbolic functional differential inclusions in Banach spaces on bounded and unbounded domains [2]-[4], using various methods, e.g. fixed point theorems.

For example [4] deals with the existence of solutions on an unbounded domain for the following hyperbolic functional differential inclusion (Darboux problem):

$$
\left\{\begin{array}{l}
\frac{\partial^{2} u(x, y)}{\partial x \partial y} \in F\left(x, y, u_{(x, y)}\right), \quad(x, y) \in J \times J=[0, \infty) \times[0, \infty), \\
u(x, y)=\phi(x, y), \quad(x, y) \in\left[-r_{1}, \infty\right) \times\left[-r_{2}, \infty\right) \backslash(0, \infty) \times(0, \infty),
\end{array}\right.
$$

where $F: J \times J \times C\left(\left[-r_{1}, 0\right] \times\left[-r_{2}, 0\right], E\right) \rightarrow 2^{E}$ is a nonempty closed, bounded and convex valued multivalued map, $\phi \in C\left(\left[-r_{1}, \infty\right) \times\left[-r_{2}, \infty\right) \backslash\right.$ $(0, \infty) \times(0, \infty), E), r_{1}>0, r_{2}>0$, and $(E,|\cdot|)$ a real separable Banach space.

For each $u \in C\left(\left[-r_{1}, \infty\right) \times\left[-r_{2}, \infty\right), E\right)$ and each $(x, y) \in J \times J$ the function $u_{(x, y)}:\left[-r_{1}, 0\right] \times\left[-r_{2}, 0\right] \rightarrow E$ is defined by $u_{(x, y)}(s, t)=u(x+s$, $y+t)$ for $(s, t) \in\left[-r_{1}, 0\right] \times\left[-r_{2}, 0\right]$. Using Ma's fixed point theorem, the authors prove that the problem has at least one solution on $\left[-r_{1}, \infty\right) \times$ $\left[-r_{2}, \infty\right)$.

Picard's problem [8] for a quasilinear hyperbolic equation consists in determining one of its solutions, provided that the values of the solution on an arc of a characteristic curve and also on another curve having a common point with the former arc are known; this common point may be taken as the origin of coordinates. Picard's problem in which one of the data carrying curves is a segment of a characteristic curve is a particular case of the classical Goursat problem [8].

In this paper, by analogy with Picard's problem for the quasilinear singlevalued hyperbolic equations [8], [23], we consider the Picard-Ionescu problem for hyperbolic inclusions with modified argument of the form

$$
\frac{\partial^{2} z(x, y)}{\partial x \partial y} \in F(x, y, z(g(x, y), h(x, y))), \quad(x, y) \in D=[0, a] \times[0, b],
$$


with initial values

$$
\left\{\begin{array}{l}
z(x, 0)=P(x), \quad 0 \leq x \leq a \\
z(\psi(y), y)=Q(y), \quad 0 \leq y \leq b
\end{array}\right.
$$

where the curve $\gamma$ is given by $x=\psi(y), \psi \in C^{1}([0, b] ;[0, a])$ is a given monotonic function, and

$$
\psi(0)=0, \quad 0 \leq \psi(y) \leq a \quad \text { for } 0 \leq y \leq b,
$$

$F: D \times \Omega \rightarrow 2^{\mathbb{R}^{n}}$ is a multifunction with compact, convex and nonempty values, $\Omega \subset \mathbb{R}^{n}$ is an open subset, and $g \in C(D ;[0, a]), h \in C(D ;[0, b])$, $P \in A C\left([0, a] ; \mathbb{R}^{n}\right), Q \in A C\left([0, b] ; \mathbb{R}^{n}\right)$ with $P(0)=Q(0)$.

Under suitable assumptions, we prove the existence of a local solution of this problem, and that the set of its solutions is compact in the Banach space $C\left(D_{0} ; \mathbb{R}^{n}\right), D_{0}=\left[0, x_{0}\right] \times\left[0, y_{0}\right] \subset D, x_{0}=\psi\left(y_{0}\right), 0 \leq y_{0} \leq b ;$ moreover as a function of the initial values, this set defines an upper-semicontinuous multifunction.

This study was suggested by papers which deal with the Picard problem [8], [11], [22], [25], with the Picard-Ionescu problem for single-valued hyperbolic equations [13], [14], [28], and by [26], [27].

2. Preliminaries. The definitions and Theorem 2.1 in this section are taken from [5], [10]-[12], [19]-[21], [24].

Definition 2.1. Let $X$ and $Y$ be two nonempty sets. A multifunction $\Phi: X \rightarrow 2^{Y}$ is a function from $X$ into the family of all nonempty subsets of $Y$. To each $x \in X, \Phi$ associates a subset $\Phi(x)$ of $Y$. The set $\bigcup_{x \in X} \Phi(x)$ is the range of $\Phi$.

Definition 2.2. Let $\Phi: X \rightarrow 2^{Y}$.

(a) If $A \subset X$, the image of $A$ under $\Phi$ is $\Phi(A)=\bigcup_{x \in A} \Phi(x)$.

(b) If $B \subset Y$, the counterimage of $B$ under $\Phi$ is

$$
\Phi^{-}(B)=\{x \in X \mid \Phi(x) \cap B \neq \emptyset\} .
$$

(c) The graph of $\Phi$, denoted by $\operatorname{graph} \Phi$, is the set

$$
\operatorname{graph} \Phi=\{(x, y) \in X \times Y \mid y \in \Phi(x)\}
$$

Definition 2.3. Let $\Phi: X \rightarrow 2^{X}$. An element $x \in X$ with $x \in \Phi(x)$ is called a fixed point of $\Phi$.

Definition 2.4. A single-valued function $\varphi: X \rightarrow Y$ is said to be a selection of $\Phi: X \rightarrow 2^{Y}$ if $\varphi(x) \in \Phi(x)$ for all $x \in X$.

Definition 2.5. Let $X$ and $Y$ be two topological spaces. A multifunction $\Phi: X \rightarrow 2^{Y}$ is upper-semicontinuous if, for any closed subset $B \subset Y$, $\Phi^{-}(B)$ is closed in $X$. 
Definition 2.6. If $(X, \mathcal{F})$ is a measurable space and $Y$ is a topological space, a multifunction $\Phi: X \rightarrow 2^{Y}$ is measurable if $\Phi^{-}(B) \in \mathcal{F}$ for every closed subset $B \subset Y$.

Theorem 2.1 ([24]). Let $X$ and $Y$ be two metric spaces, $Y$ compact, and $\Phi: X \rightarrow 2^{Y}$ a multifunction with closed values. The following assertions are equivalent:

(i) $\Phi$ is upper-semicontinuous;

(ii) the graph of $\Phi$ is closed in $X \times Y$;

(iii) for any sequences $\left(x_{n}\right)_{n \in \mathbb{N}} \subset X,\left(y_{n}\right)_{n \in \mathbb{N}} \subset Y$, from $x_{n} \rightarrow x, y_{n} \in$ $\Phi\left(x_{n}\right), y_{n} \rightarrow y$, it follows that $y \in \Phi(x)$.

Definition 2.7 ([10]-[12]). A function $u: D \rightarrow \mathbb{R}^{n}$ is absolutely continuous in Carathéodory's sense $[5, \S \S 565-570]$ if $u(x, y)$ is continuous on $D$, absolutely continuous in $x$ (for any $y$ ), absolutely continuous in $y$ (for any $x$ ), $u_{x}(x, y)$ is (possibly after a suitable definition on a two-dimensional set of zero measure) absolutely continuous in $y$ (for any $x$ ) and $u_{x y}$ is Lebesgueintegrable on $D$.

We denote the class of functions absolutely continuous in Carathéodory's sense by $C^{*}\left(D ; \mathbb{R}^{n}\right)[10]-[12]$.

We denote by $A C\left(\left[t_{1}, t_{2}\right] ; \mathbb{R}^{n}\right)$ the space of absolutely continuous functions $f:\left[t_{1}, t_{2}\right] \rightarrow \mathbb{R}^{n}$, endowed with the norm

$$
\|f\|=\sup _{t \in\left[t_{1}, t_{2}\right]}\|f(t)\|+\int_{t_{1}}^{t_{2}}\left\|f^{\prime}(t)\right\| d t .
$$

3. Results. Similarly to [6] and [26], [27] we define the notion of a $l o-$ cal solution for the Picard-Ionescu problem (1.1)-(1.2) and we prove the existence of a local solution, together with some properties of the set of all solutions, namely that it is a compact subset in the Banach space $C\left(D_{0} ; \mathbb{R}^{n}\right)$ and, as a function of initial values, it defines an upper-semicontinuous multifunction.

Let the following hypotheses be satisfied:

$\left(H_{0}\right) \quad$ The curve $\gamma: x=\psi(y), 0 \leq y \leq b$, is defined by the monotonic function $\psi \in C^{1}([0, b] ;[0, a])$ which satisfies (1.3).

$\left(H_{1}\right) \quad F: D \times \Omega \rightarrow 2^{\mathbb{R}^{n}}$ is a multifunction with compact, convex, nonempty values in $\mathbb{R}^{n}, D=[0, a] \times[0, b] \subset \mathbb{R}^{2}$ and $\Omega \subset \mathbb{R}^{n}$ is an open subset.

$\left(H_{2}\right) \quad$ For any $(x, y) \in D$, the mapping $z \mapsto F(x, y, z)$ is upper-semicontinuous on $\Omega$.

$\left(H_{3}\right) \quad$ For any $z \in \Omega$, the mapping $(x, y) \mapsto F(x, y, z)$ is Lebesgue-measurable on $D$. 
$\left(H_{4}\right) \quad g \in C(D ;[0, a])$ and $h \in C(D ;[0, b]), 0 \leq g(x, y) \leq x \leq a, 0 \leq$ $h(x, y) \leq y \leq b$.

$\left(H_{5}\right) \quad$ There exists a function $k: D \rightarrow \mathbb{R}_{+}, k \in \mathcal{L}^{1}\left(D ; \mathbb{R}_{+}\right)$, such that

$$
\|\zeta\| \leq k(x, y), \quad \forall \zeta \in F(x, y, z), \forall(x, y) \in D, \forall z \in \Omega .
$$

$\left(H_{6}\right) \quad$ The functions $P \in A C\left([0, a] ; \mathbb{R}^{n}\right), Q \in A C\left([0, b] ; \mathbb{R}^{n}\right)$ satisfy $P(0)=$ $Q(0)$.

Remark. The function $\alpha: D \rightarrow \mathbb{R}^{n}$ defined by

$$
\alpha(x, y)=P(x)+Q(y)-P(\psi(y)), \quad(x, y) \in D,
$$

is absolutely continuous in Carathéodory's sense on $D$.

Denote by $M \subset \Omega$ a convex compact nonempty set. A point $\left(x_{0}, y_{0}\right) \in$ ] $0, a] \times] 0, b], x_{0}=\psi\left(y_{0}\right)$, can be found such that:

(a) $\int_{0}^{x_{0}} \int_{0}^{y_{0}} k(u, v) d u d v<d\left(M, C_{\Omega}\right)$, since hypothesis $\left(H_{5}\right)$ ensures that the function $k$ is integrable; $d\left(M, C_{\Omega}\right)$ is the distance from $M$ to $C_{\Omega}=\mathbb{R}^{n}-\Omega$;

(b) $\alpha\left(D_{0}\right) \subseteq M$, where $\alpha: D \rightarrow \mathbb{R}^{n}$ is defined by (3.1) and $D_{0}=$ $\left[0, x_{0}\right] \times\left[0, y_{0}\right], x_{0}=\psi\left(y_{0}\right)$.

Definition 3.1. The Picard-Ionescu problem for the hyperbolic inclusion with modified argument (1.1) means to determine a solution of this inclusion which satisfies the initial conditions (1.2).

Definition 3.2. A local solution of the Picard-Ionescu problem (1.1) + (1.2) is defined as a function $Z: D_{0} \rightarrow \Omega, Z \in C^{*}\left(D_{0} ; \mathbb{R}^{n}\right)$, which is absolutely continuous in Carathéodory's sense and satisfies (1.1) for a.e. $(x, y) \in D_{0}$, and also conditions (1.2) for all $x \in\left[0, x_{0}\right]$ and all $y \in\left[0, y_{0}\right]$.

Theorem 3.1. Let the hypotheses $\left(H_{0}\right)-\left(H_{6}\right)$ be satisfied. Then:

(i) there exists a local solution $Z$ of the Picard-Ionescu problem (1.1)+ $(1.2)$;

(ii) the set $S_{\alpha}$ of local solutions is compact in $C\left(D_{0} ; \mathbb{R}^{n}\right)$;

(iii) the multifunction $\alpha \mapsto S_{\alpha}$ is upper-semicontinuous from the product $A C\left(\left[0, x_{0}\right] ; \mathbb{R}^{n}\right) \times A C\left(\left[0, y_{0}\right] ; \mathbb{R}^{n}\right), x_{0}=\psi\left(y_{0}\right)$, to $C\left(D_{0} ; \mathbb{R}^{n}\right)$.

Proof. (i) We denote by $\mathcal{Z}_{M}$ the set of functions $Z \in C^{*}\left(D_{0} ; \mathbb{R}^{n}\right)$ which satisfy

$$
\left\|\frac{\partial^{2} Z(x, y)}{\partial x \partial y}\right\| \leq k(x, y) \quad \text { for a.e. }(x, y) \in D_{0},
$$

and also conditions (1.2). The notation $\mathcal{Z}_{M}$ is suitable because $\alpha(x, y) \in M$ for $(x, y) \in D_{0}$, according to (b). We remark that the absolute continuity of $Z$ in Carathéodory's sense ensures the existence of the derivative $\partial^{2} Z(x, y) / \partial x \partial y$ for a.e. $(x, y) \in D_{0}[5, \S \S 565-570]$. We have $\mathcal{Z}_{M} \subset$ $C^{*}\left(D_{0} ; \mathbb{R}^{n}\right)$. Let us prove that for any $Z \in \mathcal{Z}_{M}$, it follows that $Z(x, y) \in \Omega$. 
Indeed, let $M(x, y), N(x=\psi(y), y), N_{0}(x=\psi(y), 0), M_{0}(x, 0),(x, y) \in$ $D$, be the vertices of the rectangle

$$
D_{0}(x, y)=\{(u, v) \mid \psi(y) \leq u \leq x, 0 \leq v \leq y\} .
$$

Integrating $\partial^{2} Z(x, y) / \partial x \partial y$ on $D_{0}(x, y)$, we obtain

$$
\begin{aligned}
\iint_{D_{0}(x, y)} \frac{\partial^{2} Z(u, v)}{\partial u \partial v} d u d v=\int_{0}^{y} d v \int_{\psi(y)}^{x} \frac{\partial^{2} Z(u, v)}{\partial u \partial v} d u \\
=\int_{0}^{y}\left[\frac{\partial Z}{\partial v}(x, v)-\frac{\partial Z}{\partial v}(\psi(y), v)\right] d v \\
=\int_{0}^{y} \frac{\partial Z}{\partial v}(x, v) d v-\int_{0}^{y} \frac{\partial Z}{\partial v}(\psi(y), v) d v \\
=Z(x, y)-Z(x, 0)-Z(\psi(y), y)+Z(\psi(y), 0) \\
=Z(x, y)-P(x)-Q(y)+P(\psi(y)) .
\end{aligned}
$$

Hence

$$
\begin{aligned}
Z(x, y) & =P(x)+Q(y)-P(\psi(y))+\iint_{D_{0}(x, y)} \frac{\partial^{2} Z(u, v)}{\partial u \partial v} d u d v \\
& =\alpha(x, y)+\iint_{D_{0}(x, y)} \frac{\partial^{2} Z(u, v)}{\partial u \partial v} d u d v .
\end{aligned}
$$

We have $D_{0}=D_{0}\left(x_{0}, y_{0}\right)=\left\{(u, v) \mid 0 \leq u \leq x_{0}=\psi\left(y_{0}\right), 0 \leq v \leq y_{0}\right\}$ and $D_{0}(x, y) \subseteq D_{0}\left(x_{0}, y_{0}\right)=D_{0}$ for $0 \leq x \leq x_{0}, 0 \leq y \leq y_{0}$.

Using (a), inequality (3.2) and (3.4) we obtain

$$
\begin{aligned}
\|Z(x, y)-\alpha(x, y)\| & =\left\|\iint_{D_{0}(x, y)} \frac{\partial^{2} Z(u, v)}{\partial u \partial v} d u d v\right\| \\
& \leq \iint_{D_{0}(x, y)}\left\|\frac{\partial^{2} Z(u, v)}{\partial u \partial v}\right\| d u d v \leq \iint_{D_{0}(x, y)} k(u, v) d u d v \\
& \leq \iint_{D_{0}} k(u, v) d u d v<d\left(M, C_{\Omega}\right) .
\end{aligned}
$$

From $\alpha(x, y) \in M$ for $(x, y) \in D_{0}$, according to (b), we have

$$
d(Z(x, y), \alpha(x, y))=\|Z(x, y)-\alpha(x, y)\|<d\left(M, C_{\Omega}\right),
$$

which shows that $Z(x, y) \in \Omega$ for $(x, y) \in D_{0}$.

We prove that the set $\mathcal{Z}_{M}$ is convex and compact in $C\left(D_{0} ; \mathbb{R}^{n}\right)$. 
Indeed, let $Z_{1}, Z_{2} \in \mathcal{Z}_{M}$ and $\lambda_{1}, \lambda_{2} \in[0,1], \lambda_{1}+\lambda_{2}=1$. We have $Z_{i} \in$ $C^{*}\left(D_{0} ; \mathbb{R}^{n}\right),\left\|\partial^{2} Z_{i}(x, y) / \partial x \partial y\right\| \leq k(x, y)$ for a.e. $(x, y) \in D_{0}, Z_{i}(x, 0)=$ $P(x)$ for $0 \leq x \leq \psi\left(y_{0}\right), Z_{i}(\psi(y), y)=Q(y)$ for $0 \leq y \leq y_{0}, i=1,2$.

It follows from the properties of functions absolutely continuous in Carathéodory's sense that $\lambda_{1} Z_{1}+\lambda_{2} Z_{2} \in C^{*}\left(D_{0} ; \mathbb{R}^{n}\right)$. Moreover,

$$
\begin{gathered}
\left\|\frac{\partial^{2}\left(\lambda_{1} Z_{1}+\lambda_{2} Z_{2}\right)(x, y)}{\partial x \partial y}\right\|=\left\|\lambda_{1} \frac{\partial^{2} Z_{1}(x, y)}{\partial x \partial y}+\lambda_{2} \frac{\partial^{2} Z_{2}(x, y)}{\partial x \partial y}\right\| \\
\leq \lambda_{1}\left\|\frac{\partial^{2} Z_{1}(x, y)}{\partial x \partial y}\right\|+\lambda_{2}\left\|\frac{\partial^{2} Z_{2}(x, y)}{\partial x \partial y}\right\| \\
\leq \lambda_{1} k(x, y)+\lambda_{2} k(x, y)=k(x, y) \quad \text { for a.e. }(x, y) \in D_{0},
\end{gathered}
$$

and

$$
\begin{aligned}
\left(\lambda_{1} Z_{1}+\lambda_{2} Z_{2}\right)(x, 0) & =\lambda_{1} Z_{1}(x, 0)+\lambda_{2} Z_{2}(x, 0) \\
& =\lambda_{1} P(x)+\lambda_{2} P(x)=P(x) \quad \text { for } 0 \leq x \leq \psi\left(y_{0}\right), \\
\left(\lambda_{1} Z_{1}+\lambda_{2} Z_{2}\right)(\psi(y), y) & =\lambda_{1} Z_{1}(\psi(y), y)+\lambda_{2} Z_{2}(\psi(y), y) \\
& =\lambda_{1} Q(y)+\lambda_{2} Q(y)=Q(y) \quad \text { for } 0 \leq y \leq y_{0},
\end{aligned}
$$

showing that $\lambda_{1} Z_{1}+\lambda_{2} Z_{2} \in \mathcal{Z}_{M}$, hence $\mathcal{Z}_{M}$ is convex.

In order to prove that $\mathcal{Z}_{M}$ is compact, according to Arzelà-Ascoli's theorem, we show that $\mathcal{Z}_{M}$ is equibounded and equicontinuous.

It follows from (3.4) and (a) that

$$
\begin{aligned}
\|Z(x, y)\| & \leq\|\alpha(x, y)\|+\iint_{D_{0}(x, y)}\left\|\frac{\partial^{2} Z(u, v)}{\partial u \partial v}\right\| d u d v \\
& \leq\|\alpha(x, y)\|+\iint_{D_{0}} k(u, v) d u d v \\
& \leq\|\alpha(x, y)\|+d\left(M, C_{\Omega}\right) \quad \text { for }(x, y) \in D_{0},
\end{aligned}
$$

hence $\mathcal{Z}_{M}$ is equibounded.

Let $h, k \in \mathbb{R}$ be such that $(x+h, y+k) \in D_{0}$. From (3.4) we have

$$
\begin{aligned}
Z(x+ & h, y+k)-Z(x, y)=[P(x+h)-P(x)] \\
& +[Q(y+k)-Q(y)]-[P(\psi(y+k))-P(\psi(y))] \\
& +\int_{0}^{y+k} d v \int_{\psi(y+k)}^{x+h} \frac{\partial^{2} Z(u, v)}{\partial u \partial v} d u-\int_{0}^{y} d v \int_{\psi(y)}^{x} \frac{\partial^{2} Z(u, v)}{\partial u \partial v} d u \\
= & {[P(x+h)-P(x)]+[Q(y+k)-Q(y)]-[P(\psi(y+k))-P(\psi(y))] }
\end{aligned}
$$




$$
\begin{aligned}
& +\int_{0}^{y} d v \int_{x}^{x+h} \frac{\partial^{2} Z(u, v)}{\partial u \partial v} d u-\int_{0}^{y} d v \int_{\psi(y)}^{\psi(y+k)} \frac{\partial^{2} Z(u, v)}{\partial u \partial v} d u \\
& +\int_{y}^{y+k} d v \int_{\psi(y+k)}^{x+h} \frac{\partial^{2} Z(u, v)}{\partial u \partial v} d u
\end{aligned}
$$

from which we obtain

$$
\begin{aligned}
\| Z(x+h, y+ & k)-Z(x, y)\|\leq\| P(x+h)-P(x)\|+\| Q(y+k)-Q(y) \| \\
& +\|P(\psi(y+k))-P(\psi(y))\|+\left\|\int_{0}^{y} d v \int_{x}^{x+h} \frac{\partial^{2} Z(u, v)}{\partial u \partial v} d u\right\| \\
& +\left\|\int_{0}^{y} d v \int_{\psi(y)}^{\psi(y+k)} \frac{\partial^{2} Z(u, v)}{\partial u \partial v} d u\right\|+\left\|\int_{y}^{y+k} d v \int_{\psi(y+k)}^{x+h} \frac{\partial^{2} Z(u, v)}{\partial u \partial v} d u\right\| .
\end{aligned}
$$

From the continuity of $P$ for $0 \leq x \leq a, Q$ and $\psi$ for $0 \leq y \leq b$ and from the absolute continuity of the integral, it follows that for every $\varepsilon>0$, there exists a $\delta(\varepsilon)>0$ such that for $h<\delta(\varepsilon), k<\delta(\varepsilon)$ each of the six terms on the right hand side above is $<\varepsilon / 6$; hence $\| Z(x+h, y+k)-$ $Z(x, y) \|<\varepsilon$, which shows that $\mathcal{Z}_{M}$ is equicontinuous, hence compact in $C\left(D_{0} ; \mathbb{R}^{n}\right)$.

We denote by $\mathcal{G}$ the set of triples $(\alpha, Z, U) \in C^{*}\left(D_{0} ; \mathbb{R}^{n}\right) \times \mathcal{Z}_{M} \times \mathcal{Z}_{M}$ such that

$$
\frac{\partial^{2} U(x, y)}{\partial x \partial y} \in F(x, y, Z(g(x, y), h(x, y))) \quad \text { for a.e. }(x, y) \in D_{0} \text {. }
$$

We now prove that, for each $\alpha \in C^{*}\left(D_{0} ; \mathbb{R}^{n}\right)$ with $\alpha(x, y) \in M$ for $(x, y) \in$ $D_{0}$, the set of pairs $(Z, U)$ such that $(\alpha, Z, U) \in \mathcal{G}$ is nonempty, and the set $\mathcal{G}$ is closed.

Indeed, let $Z \in \mathcal{Z}_{M}$. The hypotheses in Theorem 1 of [6] are satisfied for $T=D_{0}, \mu$ the Lebesgue measure on $T, U=\Omega \subset \mathbb{R}^{n}, E=\mathbb{R}^{n}$ and the multifunction $F$, due to $\left(H_{2}\right)$ and $\left(H_{3}\right)$. By that theorem, there exists a $\mu$-measurable multifunction $\Gamma: D_{0} \rightarrow 2^{\mathbb{R}^{n}}$ with compact, nonempty values such that

$$
\Gamma(x, y) \subset F(x, y, Z(g(x, y), h(x, y))), \quad \forall(x, y) \in D_{0} .
$$

The hypotheses in Theorems 2 and 3 of [7] are satisfied for $T=D_{0}$, $U=\mathbb{R}^{n}, \Gamma: D_{0} \rightarrow \operatorname{Comp}\left(\mathbb{R}^{n}\right)$. Hence, there exists a measurable selection $\beta$ of $\Gamma$, i.e. a measurable single-valued function $\beta: D_{0} \rightarrow \mathbb{R}^{n}$ with $\beta(x, y) \in$ $\Gamma(x, y)$ for $(x, y) \in D_{0}$. 
Define $U: D_{0} \rightarrow \mathbb{R}^{n}$ by

$$
\begin{aligned}
U(x, y) & =\alpha(x, y)+\iint_{D_{0}(x, y)} \beta(u, v) d u d v \\
& =\alpha(x, y)+\int_{0}^{y} d v \int_{\psi(y)}^{x} \beta(u, v) d u, \quad(x, y) \in D_{0} .
\end{aligned}
$$

Then $(\alpha, Z, U) \in \mathcal{G}$ because

$$
\beta(x, y) \in \Gamma(x, y) \subset F(x, y, Z(g(x, y), h(x, y)))
$$

for a.e. $(x, y) \in D_{0}$,

$$
\begin{aligned}
& \frac{\partial^{2} U(x, y)}{\partial x \partial y}=\beta(x, y) \in \Gamma(x, y) \\
& \quad \subset F(x, y, Z(g(x, y), h(x, y))), \quad \text { for a.e. }(x, y) \in D_{0}, \\
& \left\|\frac{\partial^{2} U(x, y)}{\partial x \partial y}\right\|=\|\beta(x, y)\| \leq k(x, y), \quad \forall(x, y) \in D_{0},
\end{aligned}
$$

by hypothesis $\left(H_{5}\right)$ for $\zeta=\beta(x, y)$, and

$$
\left\{\begin{array}{l}
U(x, 0)=P(x), \quad 0 \leq x \leq x_{0}=\psi\left(y_{0}\right), \\
U(\psi(y), y)=Q(y), \quad 0 \leq y \leq y_{0} .
\end{array}\right.
$$

The conditions (3.12), which show that $U$ satisfies the initial conditions (1.2), follow from (3.8). Indeed, for $y=0$, by (3.8) we obtain

$$
U(x, 0)=\alpha(x, 0)=P(x)+Q(0)-P(\psi(0))=P(x)+Q(0)-P(0)=P(x)
$$

for $0 \leq x \leq x_{0}=\psi\left(y_{0}\right)$, and for $x=\psi(y), 0 \leq y \leq y_{0}$, (3.8) yields

$$
U(\psi(y), y)=\alpha(\psi(y), y)=P(\psi(y))+Q(y)-P(\psi(y))=Q(y), \quad 0 \leq y \leq y_{0} .
$$

To prove that $\mathcal{G}$ is closed, let $\left\{\left(\alpha_{n}, Z_{n}, U_{n}\right)\right\}_{n \in \mathbb{N}} \subset \mathcal{G}$ be a sequence convergent to $(\alpha, Z, U)$ in the space $\left(A C\left(\left[0, x_{0}\right] ; \mathbb{R}^{n}\right) \times A C\left(\left[0, y_{0}\right] ; \mathbb{R}^{n}\right)\right) \times$ $C\left(D_{0} ; \mathbb{R}^{n}\right) \times L^{1}\left(D_{0} ; \mathbb{R}^{n}\right), x_{0}=\psi\left(y_{0}\right)$. We must check that $(\alpha, Z, U) \in \mathcal{G}$.

The set $\left\{\partial^{2} U_{n}(x, y) / \partial x \partial y\right\}_{n \in \mathbb{N}}$ is relatively weakly compact in $L^{1}\left(D_{0} ; \mathbb{R}^{n}\right)$ by the Dunford-Pettis criterion [15]. Indeed, the hypotheses of the criterion are satisfied, because:

1) $\iint_{D_{0}}\left\|\frac{\partial^{2} U_{n}(u, v)}{\partial u \partial v}\right\| d u d v \leq \iint_{D_{0}} k(u, v) d u d v=K$,

$K>0$ is a constant,

2) $\iint_{A}\left\|\frac{\partial^{2} U_{n}(u, v)}{\partial u \partial v}\right\| d u d v \leq \iint_{A} k(u, v) d u d v<\varepsilon \quad$ if $\mu(A)<\delta(\varepsilon)$,

from the absolute continuity of Lebesgue integral, 
3) for every $\varepsilon>0$ there exists a compact set $C \subset D_{0}$ such that

$$
\iint_{D_{0}-C}\left\|\frac{\partial^{2} U_{n}(u, v)}{\partial u \partial v}\right\| d u d v \leq \varepsilon .
$$

It follows that $\left\{\partial^{2} U_{n}(x, y) / \partial x \partial y\right\}_{n \in \mathbb{N}}$ is weakly convergent to a function $V \in L^{1}\left(D_{0} ; \mathbb{R}^{n}\right)$. For each $(x, y) \in D_{0}$, we have

$$
\begin{aligned}
& U(x, y)=w-\lim _{n \rightarrow \infty} U_{n}(x, y) \\
& =w-\lim _{n \rightarrow \infty}\left[\alpha_{n}(x, y)+\iint_{D_{0}(x, y)} \frac{\partial^{2} U_{n}(u, v)}{\partial u \partial v} d u d v\right] \\
& \quad=\alpha(x, y)+\iint_{D_{0}(x, y)} V(u, v) d u d v=\alpha(x, y)+\int_{0}^{y} d v \int_{\psi(y)}^{x} V(u, v) d u .
\end{aligned}
$$

From the weak convergence $\partial^{2} U_{n}(x, y) / \partial x \partial y \rightarrow V(x, y),(x, y) \in D_{0}$, using a corollary of Mazur's theorem [16], it follows that there exists a sequence of convex combinations $\left\{W_{r}\right\}_{r \in \mathbb{N}}$ of the set $\left\{\partial^{2} U_{r} / \partial x \partial y, \partial^{2} U_{r+1} / \partial x \partial y, \ldots\right\}$, strongly convergent to $V$ in $L^{1}\left(D_{0} ; \mathbb{R}^{n}\right)$. Then we can extract a subsequence $\left\{W_{r_{i}}\right\}$ from $\left\{W_{r}\right\}_{r \in \mathbb{N}}$ which converges to $V$ for a.e. $(x, y) \in D_{0}$.

Since $F(x, y, Z)$ is convex and compact for all $(x, y) \in D$ and for all $Z \in \Omega$, from the previous results and from Lemma 2 of [6] we deduce that

$$
\begin{aligned}
V(x, y) & \in \bigcap_{r=1}^{\infty} \operatorname{conv} \overline{\left(\bigcup_{n=r}^{\infty} \frac{\partial^{2} U_{n}(x, y)}{\partial x \partial y}\right)} \\
& \subset \bigcap_{r=1}^{\infty} \operatorname{conv} \overline{\left(\bigcup_{n=r}^{\infty} F\left(x, y, Z_{n}(g(x, y), h(x, y))\right)\right)} \\
& \subset F(x, y, Z(g(x, y), h(x, y))) \quad \text { for a.e. }(x, y) \in D_{0} .
\end{aligned}
$$

Since $\partial^{2} U(x, y) / \partial x \partial y=V(x, y)$ by (3.13), it follows from (3.14) that

$$
\frac{\partial^{2} U(x, y)}{\partial x \partial y}=V(x, y) \in F(x, y, Z(g(x, y), h(x, y)))
$$

for a.e. $(x, y) \in D_{0}$, and also (3.12), hence $U$ satisfies the initial conditions (1.2) for $(x, y) \in D_{0}$, i.e. $(\alpha, Z, U) \in \mathcal{G}$.

Take $\alpha \in C^{*}\left(D_{0} ; \mathbb{R}^{n}\right)$ with $\alpha(x, y) \in M$ for $(x, y) \in D_{0}$. To each $Z \in \mathcal{Z}_{M}$ we associate the set $\Phi(Z) \subset \mathcal{Z}_{M}$ as follows:

$$
\begin{aligned}
U \in \Phi(Z) \Leftrightarrow & U \in \mathcal{Z}_{M}, \frac{\partial^{2} U(x, y)}{\partial x \partial y} \in F(x, y, Z(g(x, y), h(x, y))) \\
& \text { for a.e. }(x, y) \in D_{0} .
\end{aligned}
$$

We thus define a multifunction $\Phi: \mathcal{Z}_{M} \rightarrow 2^{\mathcal{Z}_{M}}$. The set $\Phi(Z)$ is convex, compact and nonempty. Indeed, $\Phi(Z)$ is convex since $F(x, y, Z(x, y))$ is convex 
by hypothesis $\left(H_{1}\right)$. Indeed, let $U_{i} \in \Phi(Z), i=1,2$. By definition, $U_{i} \in \mathcal{Z}_{M}$, hence $U_{i} \in C^{*}\left(D_{0} ; \mathbb{R}^{n}\right)$,

$$
\begin{gathered}
\frac{\partial^{2} U_{i}(x, y)}{\partial u \partial v} \in F(x, y, Z(g(x, y), h(x, y))) \quad \text { for a.e. }(x, y) \in D_{0}, \\
\left\|\frac{\partial^{2} U_{i}(x, y)}{\partial u \partial v}\right\| \leq k(x, y) \quad \text { for a.e. }(x, y) \in D_{0} \\
U_{i}(x, 0)=P(x) \quad \text { for } 0 \leq x \leq \psi\left(y_{0}\right), \\
U_{i}(\psi(y), y)=Q(y) \quad \text { for } 0 \leq y \leq y_{0}, \text { for } i=1,2 .
\end{gathered}
$$

For $0 \leq \lambda_{1} \leq 1,0 \leq \lambda_{2} \leq 1$ with $\lambda_{1}+\lambda_{2}=1$, we have $\lambda_{1} U_{1}+\lambda_{2} U_{2} \in \mathcal{Z}_{M}$ because $\mathcal{Z}_{M}$ is convex,

$$
\begin{aligned}
\frac{\partial^{2}\left(\lambda_{1} U_{1}+\lambda_{2} U_{2}\right)(x, y)}{\partial x \partial y} & =\lambda_{1} \frac{\partial^{2} U_{1}(x, y)}{\partial x \partial y}+\lambda_{2} \frac{\partial^{2} U_{2}(x, y)}{\partial x \partial y} \\
& \in F(x, y, Z(g(x, y), h(x, y)))
\end{aligned}
$$

for a.e. $(x, y) \in D_{0}$, because $F(x, y, Z(g(x, y), h(x, y))$ is convex by hypothesis, and

$$
\begin{gathered}
\left\|\frac{\partial^{2}\left(\lambda_{1} U_{1}+\lambda_{2} U_{2}\right)(x, y)}{\partial x \partial y}\right\| \leq \lambda_{1}\left\|\frac{\partial^{2} U_{1}(x, y)}{\partial x \partial y}\right\|+\lambda_{2}\left\|\frac{\partial^{2} U_{2}(x, y)}{\partial x \partial y}\right\| \\
\leq \lambda_{1} k(x, y)+\lambda_{2} k(x, y)=k(x, y), \text { for a.e. }(x, y) \in D_{0}, \\
\left(\lambda_{1} U_{1}+\lambda_{2} U_{2}\right)(x, 0)=\lambda_{1} U_{1}(x, 0)+\lambda_{2} U_{2}(x, 0) \\
=\lambda_{1} P(x)+\lambda_{2} P(x)=P(x) \quad \text { for } 0 \leq x \leq \psi\left(y_{0}\right), \\
\left(\lambda_{1} U_{1}+\lambda_{2} U_{2}\right)(\psi(y), y)=\lambda_{1} U_{1}(\psi(y), y)+\lambda_{2} U_{2}(\psi(y), y) \\
=\lambda_{1} Q(y)+\lambda_{2} Q(y)=Q(y) \quad \text { for } 0 \leq y \leq y_{0} .
\end{gathered}
$$

Hence $\Phi(Z)$ is convex. We have $\Phi(Z) \subset \mathcal{Z}_{M}$ where $\mathcal{Z}_{M}$ is compact. The multifunction $\Phi$ has a closed graph, because graph $\Phi=\mathcal{G}$ for each fixed $\alpha$ and $\mathcal{G}$ is closed. It follows that $\Phi(Z)$ is compact in $C\left(D_{0} ; \mathbb{R}^{n}\right)$. The set $\Phi(Z)$ is nonempty since it contains $U$, defined by (3.8).

The multifunction $\Phi: \mathcal{Z}_{M} \rightarrow 2^{\mathcal{Z}_{M}}$, having a closed graph, is uppersemicontinuous by Theorem 2.1. By the Kakutani-Ky Fan fixed point theorem [15], [24], $\Phi$ has a fixed point, i.e. there exists $Z \in \mathcal{Z}_{M}$ such that $Z \in \Phi(Z)$, hence $Z=U$; but $U$ is of the form (3.8), so $Z$ is a solution of the Darboux-Ionescu problem (1.1)+(1.2).

(ii) $S_{\alpha}$ is compact because it is the set of fixed points of the multifunction $\Phi$.

(iii) The graph $\mathcal{H}$ of the multifunction $\alpha \mapsto S_{\alpha}$, defined on $C^{*}\left(D_{0} ; \mathbb{R}^{n}\right)$ with values in $2^{\mathcal{Z}_{M}}, S_{\alpha} \subset \Phi\left(\mathcal{Z}_{M}\right) \subset 2^{\mathcal{Z}_{M}}$, is closed in $\left(A C\left(\left[0, x_{0}\right] ; \mathbb{R}^{n}\right) \times\right.$ $\left.A C\left(\left[0, y_{0}\right] ; \mathbb{R}^{n}\right)\right) \times \mathcal{Z}_{M}, x_{0}=\psi\left(y_{0}\right)$, since $\mathcal{H}$ is the image of the compact set 
$\mathcal{H}_{1}$ of the triples $(\alpha, Z, U) \in \mathcal{G}$ with $Z=U$ under the projection mapping $(\alpha, Z, U) \mapsto(\alpha, Z)$. The mapping $\alpha \mapsto S_{\alpha}$ is - in general-a multifunction because several solutions of problem $(1.1)+(1.2)$ can exist, which are fixed points of the mapping $\Phi$ corresponding to the same function $\alpha$. Because $\mathcal{H}$ is closed by Theorem 2.1, it follows that $\alpha \mapsto S_{\alpha}$ is upper-semicontinuous on $A C\left(\left[0, x_{0}\right] ; \mathbb{R}^{n}\right) \times A C\left(\left[0, y_{0}\right] ; \mathbb{R}^{n}\right), x_{0}=\psi\left(y_{0}\right)$, which completes the proof.

REMARKs. (a) The same method yields the existence of a local solution to the Picard-Ionescu problem $(1.1)+\left(1.2^{\prime}\right)$, where

$$
\left\{\begin{array}{l}
z(x, 0)=P(x), \quad-a^{\prime} \leq x \leq a \\
z(\psi(y), y)=Q(y), \quad 0 \leq y \leq b
\end{array}\right.
$$

where $P \in A C\left(\left[-a^{\prime}, a\right] ; \mathbb{R}^{n}\right), Q$ and $\psi$ satisfy the same hypotheses as before, $x=\psi(y)$ takes values in $\left[-a^{\prime}, a\right]$ with $a^{\prime}>0$.

(b) In a similar way, one can prove the existence of a local solution of the Picard-Ionescu problem $(1.1)+\left(1.2^{\prime \prime}\right)$, where

$$
\left\{\begin{array}{l}
z(x, 0)=P(x), \quad-a^{\prime} \leq x \leq a, \\
z(\psi(y), y)=Q(y), \quad-b^{\prime} \leq y \leq b,
\end{array}\right.
$$

where $P \in A C\left(\left[-a^{\prime}, a\right] ; \mathbb{R}^{n}\right), Q \in A C\left(\left[-b^{\prime}, b\right] ; \mathbb{R}^{n}\right), P(0)=Q(0)$, and the function $\psi \in C^{1}\left(\left[-b^{\prime}, b\right] ;\left[-a^{\prime}, a\right]\right)$ satisfies

$$
\psi(0)=0, \quad-a^{\prime} \leq \psi(y) \leq a \quad \text { for }-b^{\prime} \leq y \leq b, \text { with } b^{\prime}>0 .
$$

\section{References}

[1] M. Benchohra and S. K. Ntouyas, An existence theorem for an hyperbolic differential inclusion in Banach space, Discuss. Math. Differ. Incl. 22 (2002), 5-16.

[2] - - - On an hyperbolic functional differential inclusion in Banach space, Fasciculi Math. 33 (2002), 27-35.

[3] - - - Hyperbolic functional differential inclussions in Banach space with nonlocal conditions, Funct. Approx. Comment. Math. 29 (2001), 29-39.

[4] - - - An existence result for hyperbolic functional differential inclusions, Comment. Math. Prace Mat. 42 (2002), 1-16.

[5] C. Carathéodory, Vorlesungen über reelle Funktionen, 3rd ed., Chelsea, New York, 1968.

[6] C. Castaing, Sur les équations différentielles multivoques, C. R. Acad. Sci. Paris Sér. A 263 (1966), 63-66.

[7] —, Quelques problèmes de mésurabilité liés à la théorie de la commande, ibid. 262 (1966), 409-411.

[8] M. Cinquini-Cibrario and S. Cinquini, Equazioni a derivate parziali di tipo iperbolico, Ed. Cremonese, Roma, 1964. 
[9] M. Dawidowski, M. Kisielewicz and I. Kubiaczyk, Existence theorem for hyperbolic differential inclusion with Carathéodory right hand side, Discuss. Math. Differ. Incl. 10 (1990), 69-75.

[10] K. Deimling, A Carathéodory theory for systems of integral equations, Ann. Mat. Pura Appl. (4) 86 (1970), 217-260.

[11] - Das Picard-Problem für $u_{x y}=f\left(x, y, u, u_{x}, u_{y}\right)$ unter Carathéodory-Voraussetzungen, Math. Z. 114 (1970), 303-312.

[12] - Das Goursat-Problem für $u_{x y}=f(x, y, u)$, Aequationes Math. 6 (1971), 303312.

[13] G. Dezsö, On the problem of Picard-Ionescu, in: Seminar on Fixed Point Theory (Cluj-Napoca, 1983/84) Preprint 84-3, "Babeş-Bolyai" Univ., 1984, 29-35.

[14] - Fixed point principles and applications in the theory of hyperbolic equations with modified argument, $\mathrm{PhD}$ thesis, Faculty of Math., "Babeş-Bolyai" Univ., ClujNapoca, 2000 (in Romanian with English abstract).

[15] R. E. Edwards, Functional Analysis. Theory and Applications, Holt, Rinehart and Winston, 1965.

[16] E. Hille and R. S. Phillips, Functional Analysis and Semigroups, Colloq. Publ. 31, Amer. Math. Soc., 1957 (fourth printing of revised edition, 1981).

[17] D. V. Ionescu, Sur une classe d'équations fonctionnelles, thèse, Paris, 1927.

[18] J. Kisyński, Sur le problème de Picard pour l'équation hyperbolique aux dérivées partielles du second ordre, Ann. Univ. M. Curie-Skłodowska Sect. A 13 (1959), $5-24$.

[19] S. Marano, Generalized solutions of partial differential inclusions depending on a parameter, Rend. Accad. Naz. Sci. XL Mem. Mat. 13 (1989), 281-295.

[20] - Classical solutions of partial differential inclusions in Banach spaces, Appl. Anal. 42 (1991), 127-143.

[21] —, Controllability of partial differential inclusions depending on a parameter and distributed parameter control processes, Matematiche (Catania) 45 (1990), 283-300.

[22] A. Pelczar, Some functional differential equations, Dissertationes Math. 100 (1973).

[23] E. Picard, Leçons sur quelques types simples d'équations aux dérivées partielles, Paris, 1927, 162-166.

[24] I. A. Rus, Principles and Applications of Fixed Point Theory, Editura Dacia, ClujNapoca, 1979 (in Romanian).

[25] G. Santagati, Sul problema di Picard in ipotesi di Carathéodory, Ann. Mat. Pura Appl. (4) 75 (1967), 47-94.

[26] G. Teodoru, A study of solutions of equations of the form $\partial^{2} Z / \partial x \partial y \in F(x, y, z)$, PhD thesis, Univ "Al. I. Cuza", Iaşi, 1984.

[27] — Le problème de Picard pour une équation aux dérivées partielles multivoque, Bul. Inst. Politehn. Iaşi Seçt. I 33 (37) (1987), 47-50.

[28] M. Zima, On an integral equation with deviated arguments, Demonstratio Math. 28 (1995), 967-973.

Department of Mathematics

Technical University "Gh. Asachi" Iaşi

11 Carol I Blvd., RO-700506 Iaşi 6, Romania

E-mail: teodoru@math.tuiasi.ro 